\title{
Significance of Social Support Among Black Mental Health Counselors: The Role of Counselor Educators and Supervisors in Combating Race-Related Stress
}

\author{
La Toya L Patterson, PhD, LCPC
}

Department of Psychology and Counseling, Chicago State University 9501 S. King Dr. Chicago, United States.

\author{
Article Details \\ Article Type: Research Article \\ Received date: $17^{\text {th }}$ March, 2021 \\ Accepted date: $08^{\text {th }}$ May, 2021 \\ Published date: $12^{\text {th }}$ May, 2021
}

"Corresponding Author: La Toya L. Patterson, PhD, LCPC, Department of Psychology and Counseling, Chicago State University 9501 S. King Dr. Chicago, United States. E-mail: 1patte25@csu.edu

Citation: Patterson, L.T.L. (2021). Significance of Social Support Among Black Mental Health Counselors: The Role of Counselor Educators and Supervisors in Combating Race-Related Stress. J Ment Health Soc Behav 3(1):140. https://doi. org/10.33790/jmhsb1100140

Copyright: (C2021, This is an open-access article distributed under the terms of the Creative Commons Attribution License 4.0, which permits unrestricted use, distribution, and reproduction in any medium, provided the original author and source are credited.

\begin{abstract}
In general, mental health counselors face unique challenges in their job roles because of the population that they work with. However, Black Americans who worked in predominately White workspaces perceived race-based discrimination to be a real issue. Perseverative cognition related to work can cause an increase in the individual's need to recovery because it maintains work-related stressors that deplete the individual's resources further. The repetitive thoughts can lead to anticipatory cognition or stress, which makes the individual hyperaware or vigilant due to thinking they may experience racial discrimination or a racial encounter. Social support has been used to buffer the adverse effects that are caused by racial encounters. Social support has shown to have an influence on mental health by buffering the effects of stressors. Participants were provided a survey that include three questionnaires to complete. The results showed that the overall models for the two analyses were significant. However, the moderators for the analyses were not significant.
\end{abstract}

Keywords: African American, Counselors, Microaggressions, Workplace, Occupational Stress, Burnout

\section{Introduction}

Currently, researchers discuss how race-related stress can have long-lasting effects; however, the current literature does not focus on the anticipatory stress or response that African Americans experience after a race-based incident. The term anticipatory stress was introduced by Utsey and colleagues in 2012[1]. Anticipatory stress is described as the experience before experiencing the stressor. When African Americans are repeat victims of a race-based incident, they will develop an anticipatory racism reaction, which is a defense mechanism for African Americans [1]. Hyperawareness and vigilance are increased because the individual was expecting racial discrimination or prejudicial behavior based on their racial identity [2].

In the workplace, African Americans that are working in predominately White workspaces consider racism to be a real issue. Lack of advancement, poor treatment, and underutilization are just some of the issues that have been linked to racial discrimination [3]. For African Americans in the workplace, structural racism plays a major role in race-based incidents. The race of the individual can shape the occupational opportunities through structural racism, beginning with policies and then "de facto practices woven into labor market institutions" [4]. Given there is an abundance of literature that examines how occupational stress and burnout are a problem for mental health counselors, several gaps within the literature still exist. Most of the literature that examines occupational stress and burnout for mental health counselors, do not discuss how race can play a role in burnout for African American mental health counselors. In addition, very few articles attempted to encapsulate the various contexts through which people of color, namely African Americans, experience burnout and race-related stress. Franklin et al. [5] shared the experience of a Black American social worker in a predominately White workspace, and how several race-based incidents resulted in burnout.

According to Lambert and Hopkins (1995), little is known about the impact that race has on workers' experience of the workplace beyond poor job conditions and strained workplace relationships. Much of the knowledge that has been obtained about workplace conditions that are connected to adverse health, safety outcomes and stress with few large African American samples [6]. In addition, some research efforts have studied the impact of workplace racial and ethnic discrimination on African Americans and other people of color; however, these studies have examined race-related stress on the outcomes on job quality and job satisfaction but not its direct impact on occupational safety and health outcomes [6].

Social support in the workplace has been associated with psychological benefits because it allows for an increase in the person's social integration, sense of belonging, self-esteem, and positive affect [7]. Among mental health practitioners and researchers, social support has been garnering a lot of attention [8]. For African Americans, having social support can assist with the development of coping strategies [9]. A trend in the research related to African Americans that reported a race-based incident showed that they perceived social support as being support, but it was also connected to less depressive symptoms and their life satisfaction increased [10]. Ford (1985)[11] reported that individuals that have higher levels of social support at work are less likely to have stress transactions with the work environment, which can result in strain and ill-health. Co-workers can provide support for each other when dealing with emotional experiences that are work-related, such as emotional labor that the workers perform for one another and feelings of anger towards another worker [7]. In studies that looked at social support and stressors at work have observed that the support that is experienced as intangible and informal, both male and female cited their supervisors and coworkers as their main support or primary resource[12]. The results of this study will fill the void in the existing 
literature and provide insight into how perseverative cognition and anticipatory cognition can play a role in burnout and how social support can weaken the association between perseverative cognition, anticipatory cognition, and burnout for Black mental health counselors.

\section{Materials and Methods \\ Sample}

To determine the sample size needed for this study, an A priori test was performed using the $\mathrm{G}^{*}$ Power 3.1.9 software [13]. The statistical parameters established for the power analysis that was used to calculate the number of required participants included: effect size $(\mathrm{f} 2=.10)$, significance level $(\alpha=.05)$, and power $(.80) .100$ participants were required for the study to achieve a medium effect. A total of 100 participants took the survey; however, 21 participants were excluded due to incomplete responses on the questionnaires and not meeting the inclusion criteria for the study. The removal of the 21 participants brought the total number of participants to 79 .

The participants consisted of 56 (70.87\%) females, 22 (27.84\%) males and $1(1.27 \%)$ transgender participate. Seventy-three $(92.40 \%)$ participants self-identified as Black/African American and 6 (7.59\%) participants self-identified as biracial with African origins. The educational background of the participants was $1(1.27 \%)$ participant has a bachelor's degree, 47 (59.49\%) participants have master's degrees, 21 (26.58\%) 59 participants have Ph.Ds., and 10 (12.66\%) participants have other degrees (i.e., Ed.D. and Psy.D). Years of practice varied among the participants from less than 1 year $(1.27 \%)$, $1-5$ years $(29.11 \%), 6-10$ years $(30.38 \%), 10-15$ years $(13.92 \%), 15-$ 20 years $(18.99 \%)$, and over 20 years $(6.33 \%)$.

\section{Instruments}

Demographic Questionnaire. Each participant completed a questionnaire that asked their age, gender, educational degree, years of practicing mental health, type of license held, the type of work setting where they spend much of their time and practicing state. The demographic questionnaire also asked about the racial makeup of staff and clients, satisfaction with support within the workplace, satisfaction with support from their supervisor/direct supervisor, participants' comfort with going to their supervisor after a racial encounter, and outside support.

Prolonged Activation and Anticipatory Race-Related Stress Scale (PARS). The initial items on the PARS were developed from the review of existing measures for race-related stress as well as a review of related literature, and review of prolonged activation of stress literature. Also, the PARS was developed because of the theoretical limitations of current measurements. The development of the PARS was made to reflect the prolonged activation stress process which includes the following: perseverative cognition of race-related stressors, secondary appraisal of race-related stressors, and anticipatory race-related stress responses. The PARS is not intended to measure all race-related stress situations; however, it is used for capturing the process of prolonged activation stress response to race-related stress in specific race-related situations [1]. The PARS is a 17-item self-report measure of prolonged activation and anticipatory race-related stress response in African Americans. There are four subscales included in the PARS that measure factors that are associated with prolonged activation and the anticipatory stress response to race-related stressors. The four subscales of the PARS are perseverative cognition scale, anticipatory race-related stress scalepsychological, anticipatory race-related stress scale-physiological, and secondary appraisal scale. The perseverative cognition scale has five items that measure the degree to which a person cognitively perseverates about a specific incident of racism. The anticipated race-related stress scale-psychological has four items that measure the degree of an individual cognitively anticipating future racerelated stressor encounters. The anticipatory race-related stress scale-physiological has four items that measure the individual's physiological reaction to the anticipated encounter with the racerelated stressors. A 7-point Likert-type scale (1= strongly disagree to $7=$ strongly agree) is used to indicate the race-related stressor response [1]. Lastly, the secondary appraisal scale has four items that measure the degree in which the individual has the resources to cope with the encountered race-related event; a 7-point Likert-type scale $(1=$ strongly disagree to $7=$ strongly agree) is used to indicate whether they felt they had the resources to cope with the race-related incident. The Cronbach's alpha correlation coefficients with $95 \%$ CI were calculated for the four subscales. The perseverative cognition scale had a coefficient alpha $.77(95 \% \mathrm{CI}=.72-.82), .70(95 \% \mathrm{CI}=$ .64-.76) for the anticipatory race-related stress scale-psychological, $.85(95 \% \mathrm{CI}=.82-.88)$ for anticipatory race-related stress scalephysiological, and $.80(95 \% \mathrm{CI}=.76-.84)$ for secondary appraisal scale (Utsey et al., 2012). The PCS correlated .29 with ARS, .41 with ABARS, and -.35 with SAS. The ARS correlated .26 with the ABARS and -.01 with the SAS and the ABARS correlated -..18 with the SAS. It can be suggested that the "PARS subscales are measuring similar but distinct, constructs related to the prolonged activation and anticipatory stress response to race-related stressors among African Americans" [1] (p. 547). Confirmatory factor analysis showed that when using a conventional significance level of .05 , the model fits the data well.

Copenhagen Burnout Inventory (CBI). The CBI [14] has three subdimensions: personal burnout, work-related burnout, and clientrelated burnout. The three sub-dimensions were designed to be applied in different domains. The personal burnout questions were designed so that all people can answer them (generic scale). When creating the personal burnout scale the authors wanted to make sure they could compare individuals no matter the occupational status (e.g., young people unemployed, early retired, pensioners, etc.). the personal burnout scale answers questions such as how tired or exhausted are you? The personal burnout dimension is described as "the degree of physical and psychological fatigue and exhaustion experienced by the person". The questions that pertain to work-related burnout assume that the individual is engaged in paid work of some kind. Work- related burnout is explained, "as the degree of the physical and psychological fatigue and exhaustion that is perceived by the person as related to his/her work". Lastly, the client-related burnout questions include the "client" or another term that may be appropriate such as student, patient, inmate, etc. (p. 196). The CBI was chosen over the Maslach Burnout Inventory (MBI) because it is believed that burnout is only applied to those individuals that work in human services and that burnout is caused by factors associated with human service work, mainly the high emotional load. Also, the questions that are on the MBI are worded in a way that can only be answered by individuals that do "people work" [14]. The authors highlighted that burnout is characterized by depersonalizing, emotional exhaustion, and reduced personal accomplishment; burnout is characterized by "the simultaneous occurrence of all three dimensions" but the MBI measures all three dimensions independently. Therefore, there is one concept but there are three independent measures. In the empirical literature, this results in the same individual being analyzed at three different levels, one for each of the three dimensions [14], (p. 194). Lastly, each dimension has its own precursors and consequences; therefore, the correspondence between concept and measure is not present [14]. The CBI was used for analyzing baseline and followup data for an ongoing Project on Burnout, Motivation, and Job Satisfaction (PUMA); PUMA is a five-year intervention study that examines employees working in the human service sector. The baseline study was comprised of 1914 participants that were from various occupational settings, which included a state psychiatric prison (196), social welfare offices of a large 64 town (379), wards in a county somatic hospital (413), psychiatric ward in the same
JMHSB, an open access journal 
county (43), institutions for severely disabled in a county (307), homecare service in the capital (284), and home care service in a provincial town (292). The response rate as $80 \%$ and $83 \%$ were women. The Cronbach's alphas for internal reliability were very high (.85-.87). There were two formats of response, the first was used to measure intensity ("very high degree" to "very low degree") and the second format was used for frequency ("always" to "never/ almost never"). It was also reported that the CBI and CBI subscales are consistent with other burnout scales. Lastly, the personal burnout scale is consistent with the burnout measure (BM) questionnaire, the work-related burnout subscale is consistent with the TSK and BM questionnaire [14]. It must be noted that for this study, higher scores on the CBI subscale means less burnout and lower scores mean higher burnout among participants.

Social Provisions Scale (SPS). The Social Provision Scale (SPS) is a 24 -item instrument that was created to measure perceived social support and assess six functions. The measure has subscales termed attachment, social integration, the reassurance of worth, reliable alliance, guidance, and opportunity for nurturance [15]. The items are related on a 4- point response scale (1= strongly disagree to strongly agree). Green et al. [16] used the SPS and found that the subscales were significantly correlated with one another $\{\mathrm{r}(129)=.32-.78\}$ and the Cronbach alpha for the 22-items was .89. The alpha coefficients for the individual scales were from .64 to .76. Wills and Shinar (2000) [15] reported that the intercorrelations were higher ranging from $\mathrm{r}=$ .55 to $r=.99$. The SPS has been used with various adult populations, from new mothers to the elderly community [15]. The SPS has also been used with other diverse populations that include public school teachers, college students, therapists, and spouses of cancer patients [17]. To evaluate the reliability of the SPS, analyses were conducted on 1792 respondents that included 1183 students from psychology courses, 303 public school teachers, and 306 nurses from a military hospital. The reliability for the total Social Provisions score was .915 , this was "estimated based on the formula for the reliability of a linear combination of scores given by Nunnaly in 1978" [18], (p. 43). The SPS is a reliable and valid measure of the social provisions. The reliabilities of the individual subscales that assess the six social provisions appear to be appropriate to use in research contexts [18]. Lastly, the construct validity of the instrument is supported by findings that concern the relationship between social provisions and measures of loneliness and interpersonal relationships[18].

After approval was received from the Institutional Review Board, the principal researcher began recruiting participants for the study. Only individuals that self-identified as Black/African American were recruited for this study via email through Counselor Education and Supervision Network Listserv (CESNET-L) and Diversegrad listserv (Diversegrad-L). The principal researcher also contacted the administrators of the Illinois Counseling Association (ICA) and Chi Sigma Iota (CSI) to get permission to post the call for participants on their general announcement board. The electronic announcement was emailed to CESNET-L and Diversegrad-L; the principal researcher also posted the electronic announcement on the general announcement board for CSI and ICA. Participants that were interested in completing the study, were instructed to click on the link within the announcement to be directed to the survey. Once directed to the study, the participant had to review the inform consent which outlined the premise of the study, risk/benefits of the study, confidentiality, compensation, and information or psychological support and the principal researcher's and dissertation committee chair's contact information. Participants were granted access to the study once they agreed to participate in the study; if the participant did not want to participate in the study were redirected to the end of the survey. After completion of the study, participants were provided a debriefing statement, and psychological resources were provided in the event a participant felt distressed after completing the study.

\section{Results}

The final sample size for the study was 79 participants. On average, the participants were $40.81(\mathrm{SD}=9.43)$ years old. Many of the participants were self-identified as Black/African American (92.4\%) and $6(7.6 \%)$ participants self-identified as biracial. The largest percentage of participants held a master's degree (59.49\%). Most of the participants were licensed professional counselors $(40.51 \%)$ and most worked in community mental health clinic (CMHC) $(30.56 \%)$. Most of the participants have between 6-10 years of experience in the mental health field. Majority of the participants hold a licensed professional counselor (LPC) licensure. On average, participants reported that they receive support at their place of employment and that the support mainly comes from their colleagues/coworkers.

\section{Research Questions, Hypotheses, and Results of Statistical Analyses}

Q1. Does social support weaken the relationship between perseverative cognition and work-related burnout among Black mental health counselors in the workplace? H01. Social support will weaken the relationship between perseverative cognition and work-related burnout among Black mental health counselors in the workplace. To examine the first hypothesis a multiple regression was performed utilizing work-related burnout as the outcome variable and social support, the interaction term (perseverative cognition X social provisions scale), and perseverative cognition were the predictor variables to determine if social support weakened the relationship between perseverative cognition and work-related burnout among Black mental health counselors. Results indicated that the interaction term (perseverative cognition $\mathrm{x}$ social support) was not significant as a predictor of work-related burnout. However, there were main effects between perseverative cognition and work-related burnout and between social support and work-related burnout. The overall model using the predictors yielded significant results, $\mathrm{F}(3,74)=$ 11.61, $\mathrm{p}=<.001 . \mathrm{R}^{2}$ for the model was .320 , and the adjusted $\mathrm{R}^{2}$ was .293, with the full model accounting for $29 \%$ of the variance for work-related burnout.

Q2. Will social support weaken the relationship between anticipatory cognition and work-related burnout among Black mental health counselors in the workplace? H02. Social support will weaken the relationship between anticipatory cognition and work-related burnout among Black mental health counselors in the workplace? To examine the second hypothesis, the predictor variables were the PARS subscale anticipatory cognition, the interaction term (anticipatory cognition X social provisions scale), and social support; the outcome variable was work-related burnout. Results indicated that the interaction term (anticipatory cognition x social support) was not significant as a predictor of work-related burnout. However, there was a main effect between anticipatory cognition and work-related burnout and the main effect between social support and work-related burnout. The overall model using the predictors yielded significant results, $\mathrm{F}(3,75)=12.42, \mathrm{p}=<.001 . \mathrm{R}^{2}$ for the model was .332 and the adjusted $\mathrm{R}^{2}$ was .305 , with the full model accounting for $30 \%$ of the variance for work-related burnout.

An ordinal interaction with work-related burnout was found, which suggested that as individuals in the lower social support group experienced work-related burnout, the more perseverative cognition they experienced. The interaction indicates that the effect of perseverative cognition on burnout is different for different levels of social support. An ordinal interaction was found with work-related burnout, which means that as individuals in the lower social support group experience more anticipatory cognition, the more work-related burnout they would experience. A similar pattern was found with individuals with higher levels of social support except when they had less anticipatory cognition, the less work-related burnout the individuals would experience. 


\section{Discussion}

The effects of perseverative cognition and anticipatory cognition have been documented in the literature and research. However, most of the studies and literature were not specific to Black mental health counselors. This study is unique because it examined different dimensions of race-related stress connection to work-related burnout for Black mental health counselors in the workplace. More specifically, the principal researcher was interested in the impact of social support, perseverative cognition, and anticipatory cognition on work-related burnout for Black mental health counselors. This study aimed to investigate how neurocognitive processes, namely, perseverative cognition and anticipatory cognition, interacted with social support to weaken the impact of work-related burnout for Black mental health counselors.

The current study's results did not support the hypotheses that social support would weaken the relationship between perseverative cognition and work-related burnout and the relationship between anticipatory cognition and work-related burnout among Black mental health counselors. Results from the first regression analysis found that there was significance in the relationship between perseverative cognition (predictor variable) and work-related burnout (outcome variable). There was also a main effect between social support and work-related burnout. However, the moderator was insignificant. The second regression model found that there was a main effect between anticipatory cognition (predictor variable) and work-related burnout (outcome variable). There was a main effect between social support and work-related burnout, but the moderator was insignificant. This suggests that constant worrying about a racial encounter or anticipating a racial encounter can have an impact on work-related burnout for Black mental health counselors and that social support can work in a buffering fashion.

The findings were noteworthy because it provided evidence that continuous thinking of a racial encounter in the workplace or anticipating a race-related incident, can be a cause of work- related stress for Black mental health counselors. Perseverative cognition is described as cognitive representations of psychological stressors that are repeated or chronic in nature and activated through persistent worrying about the past or future [19]. When individuals are faced with stressors that are out of their control, the process of perseverative cognition is common[1]. For individuals that are a part of stigmatized groups, discussing a stressor such as racism can prompt recollections that feel uncontrollable as well as stressful [20]. Perseverative cognition does not just include conscious thoughts about the stressors, but it also includes prolonged unconscious or automatic processing of information that is related to the stressor(s). In addition, perseverative cognition can also include obsessive, intrusive thoughts, and negative flashbacks [21]. It was highlighted by Utsey et al. [1] that when there is prolonged-activation of racerelated stress, it can increase the likelihood of negative psychological and physical health of African Americans.

A large part of daily stress involves worrying about future events [22]. Regarding anticipatory cognition, racism can trigger physiological reactions that are activated by the individual's anticipatory responses and reactions to socially racist stressors [23]. Anticipation is a potential stressor that can influence the stress response. Individuals that are consistently anticipating discrimination or prejudice based on their social identity can cause vigilance or hyperawareness for cues of mistreatment [2]. The findings are consistent with a previous study conducted by Himmelstein, Young, Sanchez, and Jackson [24]. Himmelstein et al. [24] study examined how responding to everyday discrimination with anticipatory vigilance and how it relates to the health of Black men and women. Individuals that are constantly anticipating discrimination experienced physiological arousal and stress [24]. Based on the results of this study and previous findings, it can be deduced that solely anticipating race-related encounters in the workplace can cause work-related burnout for Black mental health counselors.

There can be numerous reasons why the moderator was not significant in either analysis. Research that concentrated specifically on African Americans suggested that the presence of social support is not guaranteed in vulnerable populations. When there are low levels of stress, it has been suggested that social support is beneficial; however, social support may exacerbate difficulties during higher levels of stress [25]. The effectiveness of the support received is determined by the type of support available in the face of discrimination. In addition, some individuals found that social support has a direct impact on well-being but did not feel that social support buffered the effects of perceived discrimination on well-being [25].

The social support network for African Americans tend to consist of kin, therefore, the social network is "close-knit' or dense. Additionally, African Americans' social support system usually consists of individuals that are of the same race [26]. Gender differences do exist when it comes to the social support systems of African Americans. African American women's social support systems usually consist of friends and children, whereas African American men's social support systems are comprised of spouses, kin, and friends [26]. Although African American women use social support to help combat racial discrimination, it remains unclear which form of social support must take place to alleviate the adverse effects of racial discrimination [27]. Cutrona and Russell [28] developed the optimal matching model, which believes that the effectiveness of social support is better when it focuses on the specific needs of the imposed stressor. The social support that is directly aimed at addressing racist stressors could be more successful in counteracting the adverse influence of "racial discrimination on psychological health and personal outlook than more generalized forms of support" [27], (p. 3).

A study conducted by Ajrouch et al. [25], showed that the type of social support mattered in their study sampling African American women. Their study showed that emotional support did not buffer the effects of frequent discrimination on psychological distress. After experiencing a racial encounter, it may be hard to share with others. When individuals have experienced lifelong acts of discrimination, it may influence their perception of who they can trust, which can impact the social support dynamics [25].

Franklin et al. [5] reported that the ability to find mentors and professional role models for therapists of color within the mental health field is a privilege that is not available. Research has reported that a lack of social support can be dangerous. In general, having social support in the workplace can help with increasing social integration, a sense of belonging, and the individual's self-esteem. Various studies have shown that Black workers did not feel supported in their workplace. Reduced social ties in the workplace have been reported as the reason for Black workers not feeling supported in the workplace [7]. Black mental health counselors often work in occupational settings that may not have other Black peers, which minimizes Black mental health counselors' opportunity to get samerace mentors or same-race social groups [29].

For African Americans that experience racism in the workplace, social support has been associated with fewer depressive symptoms and they also report they are more satisfied with their lives [10]. What helps Black Americans cope with stress that is related to racial discrimination is their ability to draw upon the social connections that they have. When individuals have social support connections, it has been linked to higher levels of well-being, reduced negative emotional experiences, and less emotional stress [7]. When Black Americans have social support whether it is in the workplace or their personal lives, it can serve as a buffer for reducing depressive 
symptoms that are related to racial discrimination; this will occur over time.

This study had several participants that self-identified as biracial with Black/African heritage. Ali et al. [30] reported that biracial individuals experience their ethnic identity differently, depending upon the various contexts. In addition, biracial or multiracial individuals can be excluded by racial groups as well as by "ingroups", it is recommended that future research focus on the source of the discrimination that they experience. Also, it is imperative to understand how the perceived discrimination that the biracial individuals receive influences their multiracial identity. Lastly, future research should also examine their experience with race- related stress and burnout as well as if they are receiving adequate social support in the workplace. There was a low number of Black/African American males that participated in the study. Future research should focus on the in the workplace and the level of social support that they receive while in the workplace. Pitcan, Park-Taylor \& Hayslett [31] reported that Black men are laid off at rates that are inconsistent compared to White men. Also, Black men experience racial microaggressions when they are in organizations that are predominately White [31]. workplace, will help with developing interventions or strategies to improve the workspace and to hopefully reduce situations that can cause race-related stress and burnout. This research study had one participant that self-identified as Black/African American, study the experiences of transgender Black/African American mental health professionals, to see if they are receiving social support from their supervisor or colleagues and if the social support is adequate. Whitfield et al. [32] reported that workplace discrimination against the LGBTQ community is widespread and that $25 \%$ to $60 \%$ of LGBT employees reported experiencing workplace discrimination because of their sexual orientation. Employment discrimination rates are high for transgender individuals due to transphobia [32]. Pflum, Testa, Balsam, Goldblum, and Bongar [33] reported that social support is instrumental in mitigating problems that are connected to being transgendered or gender nonconforming. In addition, having social support can assist with reducing psychological distress that is related to transphobia and discrimination and helps with the use of healthy coping mechanisms.

Currently, there are several types of social support that can be offered, instrumental, informational, emotional, esteem, tangible, and effective support. Future research can focus on which social support type is most beneficial for Black mental health counselors and combating race-related stress in the workplace. The different types of social support can provide different effects on their psychological wellbeing. Lastly, this study used Qualtrics as a means of allowing participants to complete the surveys online. Allowing the surveys to be available via Qualtrics made it accessible for participants. Using both face-to-face and electronic administering surveys may possibly yield more results. Using the face-to-face method could possibly cause bias; however, it can be used to obtain responses quicker. Another recommendation would be to study whether there is a difference in burnout and race-related stress for Black mental health counselors that are not licensed versus those that are licensed, examining whether being licensed or not licensed plays a role. Lastly, future research could include SPS subscales as a predictor variable.

The literature has suggested barriers and challenges that face mental health counselors in the workplace; however, there is a scarcity in the literature when discussing factors that impact Black mental health counselors in the workplace. Black mental health counselors face unique challenges and barriers that are not experienced by their non Black counterparts. This research highlights the importance of experience of Black/African American male mental health counselors Exploring the barriers and challenges that Black men face in the transgender individual. It would be important for future research to

social support for Black mental health counselors to reduce racerelated stress and burnout. Creating a culture or environment of support by supervisors and counselor educators is important to the success and wellbeing of Black mental health counselors. Counselor educators and supervisors that are not Black/African American can take a more active approach to understand the culture or background of Black mental health counselors and to also ask about specific assistance that they may need.

Multiracial individuals often feel torn between the monoracial groups that include their racial and ethnic heritage, therefore, no monoracial identity may truly capture their social reality [34]. Hence, supervisors and counselor educators that work with supervisees that are biracial or multiracial, must know or understand biracial ethnicity identity development. In addition, the supervisor should ask questions of themselves and the supervisees about their beliefs regarding biracial identity [30]. The same goes for working with Black/African American mental health counselors. When Black mental health counselors experience racial encounters, it is important that supervisors and counselor educators validate the individuals' experience while also providing individuals with a safe space to process their feelings. Counselor educators and supervisors should not avoid the discussion of race or negate the individual's feelings. Furthermore, Black mental health counselors, if needed, should be provided with additional resources by supervisors and counselor educators. Counselor educators and supervisors should have an open discussion about race and how it can impact Black mental health counselors.

Although greater attention is being paid to diversity, there is still emerging data that supervision is not initiating consideration for multiple diversity factors, privilege, historical trauma, or oppression being addressed [35]. It is imperative to address these diversity factors and provide adequate feedback as well as training when the supervisees do not exhibit adequate competence; these are important roles of the supervisor. Within the counselor education programs, students are usually trained on racism during their diversity orientation courses, such as "multicultural counseling or social justice/advocacy courses" [36], (p. 4). For counselor educators, it is important to have courageous conversations that address diversity issues such as racism, ableism, and oppression. These conversations can be spontaneous and address topics such as oppression and privilege that may come forth in the classroom. Counselor educators should create an environment that safe for discussing issues of diversity, invite different perspectives on diversity issues/topics, and making sure to bring the courageous conversation full circle, by connecting them to the larger systems in society.

Galek et al. [37] reported that psychotherapist relied on their supervisors and coworkers for social support to prevent burnout and that social support was connected to lower levels of burnout for people that worked in human services. Counselor education programs and supervisors should diligently work to recruit 84 and retain Black mental health counselors. Having staff that is diverse and that reflects the staff at the workplace will help with providing opportunities for Black mental health to get the mentoring they need as well as have social interactions with individuals of the same race.

\section{Conclusion}

The purpose of this study was to evaluate the impact that social support has on racerelated stress and burnout among Black mental health counselors. Multiple regression analyses were used to test the hypotheses. The results of the study strived to deliver answers to the following research questions: Does social support weaken the relationship between race-related stress and work-related burnout among Black mental health counselors? and social support will weaken the relationship between anticipatory cognition and 
work-related burnout among Black mental health counselors in the workplace? After running the multiple regression analyses there were main effects between perseverative cognition and workrelated burnout and anticipatory cognition and work-related burnout. However, the moderators for both analyses were insignificant. Some Black mental health counselors may not receive adequate social support whether it be from individuals at work or in their personal lives. The type of social support received during a racial related incident will also determine the effectiveness of social support. Social support is a promising pathway to buffering the adverse effects of race-related stress because there are various forms of social support that can be used to combat racial discrimination. In closing, the findings of this study provided previously unknown information about the effects that social support can have on race-related stress and burnout for Black mental health counselors and has provided a multitude of future research pathways and implications in this area.

Conflicts of interest: The authors have no conflicts of interest to disclose.

\section{Reference}

1. Utsey, S. O., Belvet, B., Hubbard, R. R., Fischer, N. L., Opare-Henaku, A., \& Gladney, L. L. (2012). Development and validation of the prolonged activation and anticipatory racerelated stress scale. Journal of Black Psychology, 39(6), 532- 559. doi:10.1177/0095798412461808

2. Sawyer, P. J., Major, B., Casad, B. J., Townsend, S. S. M., \& Berry-Mendes, W. (2012) Discrimination and the stress response: Psychological and physiological consequences of anticipating prejudice in interethnic interactions. American Journal of Public Health, 102(5) 1020-1026. doi:10.2105/ ajph.2011.30062027

3. Mays, V. M., Coleman, L. M., \& Jackson, J. S. (1996). Perceived race-based discrimination, employment status, and job stress in a national sample of Black women: Implications for health outcomes, 1(3), 319-329. doi:10.1037//1076- 8998.1.3.31926

4. McCluney, C. L., Schmitz, L. L., Hicken, M. T., \& Sonnega, A. (2018). Structural racism in the workplace: Does perception matter for health inequalities. Social Science \& Medicine, 199, 160-114. doi:10.1016/j.socscimed.2017.05.039

5. Franklin, A. J., Boyd-Franklin, N., \& Kelly, S. (2006). Racism and invisibility: Race related stress, emotional abuse and psychological trauma for people of color. Journal of Emotional Abuse, 6(2/3), 9-30. doi:10.1003/j135v06n02_02

6. Roberts, R. (2017). Addressing occupational stress among African Americans. In F. T. Leong, D. E. Eggerth, C. Chang, M. A. Flynn, K. J. Ford, R. O. Martinez (Eds). Occupational health disparities: Improving the well-being of ethnic and racial minority workers (pp.113-131). Washington, DC: American Psychological Association. doi:10.1037/0000021-001

7. Sloan, M. M., Evenson Newhouse, R. J., \& Thompson, A. B. (2013). Counting on coworkers: Race, social support, and emotional experience on the job. Social Psychology Quarterly, 76(4), 343-372. doi:10.1177/0190272513504937

8. Griffith, J.(1985). Social support providers: Who are they? where are they met? and the relationship of network characteristics to psychological distress. Basic and Applied Social Psychology, 6(1), 41-60. doi:10.1207/s15324834basp06010_4

9. Brown, D. L. (2008). African American resiliency: Examining racial socialization and social support as protective factors. Journal of Black Psychology, 34(1), 32-48. doi: $10.1177 / 0095798407310538$
10. Prelow, H. M., Mosher, C. E., \& Bowman, M. A. (2006). Perceived racial discrimination, social support, and psychological adjustment among African American college students. Journal of Black Psychology, 32(4), 442-454. doi:10.1177/0095798406292677

11. Ford, D. L. (1985). Facets of work support and employee work outcomes: an exploratory analysis. Journal of Management, 11(3), 5-20. doi:10.1177/014920638501100302

12. Bailey, D., Wolfe, D., \& Wolfe, C. R. (1996). The contextual impact of social support across race and gender: Implications for African American women in the workplace. Journal of Black Studies, 26(3), 287-307. doi:10.1177/002193479602600304

13. Faul, F., Erdfelder, E., Buchner, A., \& Lang, A. G. (2009). Statistical power analyses using G*Power 3.1: Tests for correlation and regression analyses. Behavioral Research Methods, 41(4), 1149-1160. doi:10.3758/brm.41.4.114925

14. Kristensen, T. S., Borritz, M., Villadsen, E. \& Christensen, K. B. (2005). The Copenhagen burnout inventory: A new tool for the assessment of burnout. Work \& Stress, 19(3), 192-207. doi:10.1080/02678370500297720

15. Wills, T., \& Shinar, O. (2000). Measuring perceived and received social support. In S. Cohen, L. G. Underwood, B. H. Gottlieb (Eds.), Social support measurement and intervention (pp. 86-2828 136). New York, NY: Oxford University Press. doi:10.1093/med:psych/9780195126709.001.0001

16. Green, B. L., Furrer, C., \& McAllister, C. (2007). How do relationships support parenting? Effects of attachment style and social support on parenting behavior in an at-risk population. American Journal of Community, 40(1-2), 96-108. doi:10.1007/ s10464-007-9187-y

17. Perera, M., Papa, N., Christidis, D., Wetherell, D., Hofman, M.S., Murphy, D.G., Bolton, D., Lawrentschuk, N. (2016). Sensitivity, Specificity, and Predictors of Positive 68 GaProstate-specific Membrane Antigen Positron Emission Tomography in Advanced Prostate Cancer: A Systematic Review and Meta-analysis, 70(6):926-937. doi: 10.1016/j. eururo.2016.06.021.

18. Cutrona, C. E., \& Russell, D. W. (1987). The provisions of social relationships and adaptation to stress. In W. H. Jones \& D. Perlman (Eds.), Advances in personal relationships (pp.3767). Greenwich, CT: JAI Press. 24

19. Foucreault, A., Menard, J., \& Stevens, C. (2016). The role of emotional support from family and neuroticism. International Journal of Psychological Studies,8(4), 77- 90. doi:10.5539/ ijps.8n4p77

20. Brondolo, E., Brady, N., Pencille, M., Beatty, D., \& Contrada, R. J. (2009). Coping with racism: A selective review of the literature and a theoretical and methodological critique. Journal of Behavioral Medicine, 32(1), 64-68. doi:10.1007/s10865-0089193-0

21. Verkuil, B., Brosschot, J. F., Gebhardt, W. A., \& Thayer, J. F. (2010). When worries make you sick: A review of perseverative cognition, the default stress response and somatic health. Journal of Experimental Psychopathology, 1(1), 87-118. doi: 10.5127 jep.009110

22. Thayer, J. F., \& Brosschot, J. F. (2010). Stress, health, and illness: The effects of prolonged physiological activity and perseverative cognition. In D. French, K. Vedhara, A. A. Kaptein, \& J. Weinman (Eds.), Health psychology (pp. 247258). Malden, MA: Blackwell Publishing Ltd.

23. Harrell, C.J.P., Burford T.I., Cage B.N., Nelson T.M., Shearon, S., Thompson A., and Green, S. (2011). Multiple Pathways Linking Racism to Health Outcomes. Du Bois Rev, 15; 8(1): 143-157. doi: 10.1017/S1742058X11000178 
24. Himmelstein, M. S., Young, D. M., Sanchez, D. T., \& Jackson, J. S. (2015). Vigilance in the discrimination-stress model for Black Americans. Psychology Health, 30(3), 253-267. doi:10. 1080/08870446.2014.966104

25. Ajrouch, K. J., Reisine, S., Lim, S., Sohn, W., \& Ismail, A. (2010). Perceived everyday discrimination and psychological distress: Does social support matter? Ethnicity \& Health, 15(4), 417-434. doi:10.1080/13557858.2000.484050

26. Hood, S., Golembiewski, E., Benbow, K., Sow, H., \& Sanders Thompson, V. (2017). Who can I turn to? Emotional support availability in African American social networks. Social Sciences, 6(3), 104-121. doi:10.3390/socsci6030104

27. Seawell, A. H., Cutrona, C., \& Russell, D. (2014). The effects of general social support and social support for racial discrimination on African Americans women's well being. Journal of Black Psychology, 40(1), 3-26. doi:10.1177/0095798412469227

28. Cutrona, C. E., \& Russell, D. W. (1990). Type of social support and specific stress: Toward a theory of optimal matching. In B. R. Sarason, I. G. Sarason, \& G. R. Pierce (Eds.), Wiley series on personality processes. Social support: An interactional view (p. 319-366)

29. Garrett, W. B. (2002). Occupational stress and job satisfaction among African American mental health professionals (Doctoral dissertation). Retrieved from ProQuest Dissertations \& Theses Global: The Sciences and Engineering Collection. (Order No. 3062537).

30. Ali, S. R., Flojo, J. R., Chronister, K. M., Hayashino, D., Smiling, Q. R., Torres, D., \& Hawley McWhirter, E. (2004). When racism is reversed: Therapists of color speak about their experiences with racism from clients, supervisees, and supervisors. In M. Rastogi \& E. Weiling (Eds.), First person accounts of minority therapist (pp. 117-134). Thousand Oaks, CA: Sage Publications, Inc.

31. Pitcan, M., Park-Taylor, J., \& Hayslett, J. (2018). Black men and racial microaggressions at work. The Career Development Quarterly, 66, 300-315. doi:10.1002/cdq.12152
32. Whitfield, D. L., Eugene Walls, N., Langenderfer-Magruder, L., \& Clark, B. (2014). Queer is the new Black? Not so much: Racial disparities in anti-LGBTQ discrimination. Journal of Gay \& Lesbian Social Services, 26(4), 426-440. doi:10.1080/1 0538720.2014 .955556

33. Pflum, S. R., Testa, R. J., Balsam, K. F., Goldblum, P. B., \& Bongar, B. (2015). Social support, trans community connectedness, and mental health symptoms among transgender and gender nonconforming adults. Psychology of Sexual Orientation and Gender Diversity, 2(3), 281-286. doi:10.1037/ $\operatorname{sgd} 0000122$

34. Giamo, L. S., Schmitt, M. T., \& Outten Robert, H. (2012). Perceived discrimination, group identification, and life satisfaction, among multiracial people: A test of the rejectionidentification model. Cultural Diversity and Ethnic Minority Psychology, 18(4), 319-328. doi:10.1037/a0029729

35. Falender, C. A., \& Shafranske, E. P. (2014). Clinical supervision: The state of the art. Journal of Clinical Psychology, 70(11), 1030-1041. doi:10.1002/jclp.22124

36. An, Z., Compton,O.C., Putz, K.W., Brinson, C., Nguyen, S.T. (2011). Bio-Inspired Borate Cross-Linking in Ultra-Stiff Graphene Oxide Thin Films,23(33). https://doi.org/10.1002/ adma.201101544

37. Galek, K., Flannelly, K. J., Greene, P. B., \& Kudler, T. (2011). Burnout, secondary traumatic stress, and social support. Pastoral Psychology, 60(5), 633-649. doi:10.1007/s11089-0110346-7. 